\title{
DESCRIÇÃO DAS PRÁTICAS DOS ENFERMEIROS DAATENÇÃO BÁSICA DIRECIONADAS PARA IDOSOS DIABÉTICOS
}

\author{
Patrícia Silva Nunes ${ }^{1}$, Marilia Braga Marques², Ana Larissa Gomes Machado ${ }^{3}$, Maria Josefina da Silva ${ }^{4}$
}

\begin{abstract}
RESUMO: Estudo transversal de caráter descritivo, realizado em Unidades Básicas de Saúde da Família de Fortaleza-CE. Participaram do estudo 42 enfermeiros, escolhidos por conveniência, convidados a participar do estudo nos dias do comparecimento dos pesquisadores às unidades de saúde, as quais foram selecionadas por amostra aleatória simples. Objetivou-se descrever as ações que o enfermeiro realiza junto aos idosos diabéticos e compará-las a partir das normas editadas pelo Ministério da Saúde. Utilizou-se para coleta de dados um questionário e observação não participante. Observou-se que as ações preconizadas pelo Ministério da Saúde são postas em prática pelos participantes do estudo, porém de forma limitada. Acredita-se que através da parceria entre cursos de graduação e pós-graduação na área gerontológica, gestores, enfermeiros e demais envolvidos na promoção da saúde dos idosos diabéticos, seja possível ampliar e qualificar as ações de cuidado a essa clientela, de modo que se alcance a integralidade da assistência.
\end{abstract}

PALAVRAS-CHAVE: Enfermagem; Diabetes mellitus; Idoso.

\section{DESCRIPTION OF THE NURSES' PRIMARY CARE PRACTICE RELATED TO DIABETICS ELDERLY}

\begin{abstract}
Cross-sectional descriptive study conducted in the Family Health Basic Units in Fortaleza/CE. Study participants were 42 nurses chosen by convenience, invited to participate in the study in the days that the researchers would be at the health units, which were selected by simple random sample. We aimed to describe the actions the nurse performs with the diabetic elderly patients and compare them from the rules issued by the Ministry of Health. A questionnaire and non-participant observation were used for data collection. It was observed that the actions recommended by the Ministry of Health are implemented by the study participants, but in a limited way. It is believed that through the partnership between undergraduate and postgraduate training in gerontology, managers, nurses and others involved in promoting the health of diabetics elderly, it is possible to expand and qualify the actions of care to this clientele, so that we can reach the full range of assistance.
\end{abstract}

KEYWORDS: Nursing; Diabetes mellitus; Aged.

\section{DESCRIPCIÓN DE LAS PRÁCTICAS DE LOS ENFERMEROS DE LAATENCIÓN BÁSI- CADIRIGIDAA DIABÉTICOS ANCIANOS}

RESUMEN: Estudio transversal de carácter descriptivo, realizado en las Unidades Básicas de Salud de la Familia de Fortaleza/CE/Brasil. Participaron del estudio 42 enfermeros, escogidos por conveniencia, invitados a participar del estudio en los días de la presencia de los investigadores a las unidades de salud, los cuales fueron seleccionados mediante muestra aleatoria simple. El objetivo fue describir las acciones que el enfermero realiza junto a los ancianos diabéticos y compararlas a partir de las normas editadas por el Ministerio de Salud. Fue utilizado para la colecta de datos un cuestionario y la observación no participante. Se observó que las acciones preconizadas por el Ministerio de Salud son ejecutadas por los participantes del estudio, pero de forma limitada. Se cree que a través de la colaboración entre los cursos de graduación y de postgraduación en el área gerontología, administradores, enfermeros y demás envueltos en la promoción de la salud de los ancianos diabéticos, sea posible ampliar y cualificar las acciones de cuidado a estos clientes, de modo que se alcance la integralidad de la asistencia.

PALABRAS CLAVE: Enfermería; Diabetes mellitus; Anciano.

\footnotetext{
${ }^{1}$ Enfermeira. Graduada pela Universidade Federal do Ceará-UFC.

${ }^{2}$ Enfermeira. Mestranda em Enfermagem pela UFC. Bolsista da Coordenação de Aperfeiçoamento de Pessoal de Nível Superior-CAPES. ${ }^{3}$ Enfermeira. Mestre em Cuidados Clínicos pela Universidade Estadual do Ceará-UECE. Docente da UECE.

${ }^{4}$ Enfermeira. Doutora em Enfermagem pela UFC. Professora Adjunto do Departamento de Enfermagem da UFC.
}

Autor correspondente:

Patrícia Silva Nunes

Universidade Federal do Ceará

Av. Eduardo Girão, 355 - 60414-540 - Fortaleza-CE, Brasil

Recebido: 27/06/09

E-mail: pat_sn@hotmail.com

Aprovado: 29/10/09

Cogitare Enferm 2009 Out/Dez; 14(4):682-8 


\section{INTRODUÇÃO}

Os idosos representam significativa parcela da população brasileira que necessita de cuidados de promoção da saúde, de prevenção de agravos e de reabilitação. Essa constatação pode ser atribuída à maior incidência de doenças crônicas nesse grupo de pessoas, cujo crescimento demográfico, nem sempre é acompanhado pelo aumento no desenvolvimento dos meios adequados para que o idoso possa cuidar de sua saúde ${ }^{(1)}$.

O crescimento populacional de idosos implica no aumento das demandas de saúde desse grupo aos serviços públicos, devido às suas particularidades no campo da assistência social e da saúde, e levanta questões fundamentais para os profissionais que cuidam dessa população.

O enfermeiro, na perspectiva de superação desses impasses e desafios, constitui-se como um profissional apto a colaborar na formulação de políticas públicas, na transformação do modelo tecnoassistencial em saúde, na reorganização dos serviços de saúde e na adequação da educação dos profissionais ${ }^{(2)}$.

O Ministério da Saúde do Brasil, considerando relevante esse aumento da população idosa em nosso País e a necessidade do setor saúde dispor de uma política devidamente expressa relacionada à saúde dessa população, aprovou em dezembro de 1999 a Política Nacional de Saúde do Idoso.

A Política tem como propósito basilar a promoção do envelhecimento saudável, a manutenção e a melhoria, ao máximo, da capacidade funcional dos idosos, a prevenção de doenças, a recuperação da saúde dos que adoecem e a reabilitação daqueles que venham a ter a sua capacidade funcional restringida, de modo a garantirlhes permanência no meio em que vivem, exercendo de forma independente suas funções na sociedade ${ }^{(3: 20-24)}$.

O envelhecimento é um processo que provoca alterações e desgastes em vários sistemas funcionais, que ocorrem de forma progressiva e irreversível. Geralmente essas alterações resultam no desenvolvimento de doenças crônicas, também chamadas de agravos não transmissíveis, entre elas o Diabetes Mellitus-DM.

Conforme estabelecido pelas Diretrizes da Sociedade Brasileira de Diabetes, o DM não é uma única doença, mas um grupo heterogêneo de distúrbios metabólicos que apresentam em comum a hiperglicemia. Essa hiperglicemia é o resultado de defeitos na ação da insulina, na secreção de insulina ou em ambos ${ }^{(4)}$.

Em nosso estudo daremos destaque ao Diabetes Mellitus Tipo 2-DM2, o qual se caracteriza por apresentar graus variáveis de resistência à insulina e deficiência relativa de secreção de insulina. O diagnóstico, na maioria dos casos, é feito a partir dos 40 anos de idade, embora possa ocorrer mais cedo, mais raramente em adolescentes ${ }^{(4)}$.

Entre os idosos, a prevalência de DM2 é maior, devido em parte, ao próprio processo de envelhecimento, "adicionalmente, alterações no metabolismo dos carboidratos ligados à idade parecem contribuir para o desenvolvimento, uma vez que valores glicêmicos tendem a ser mais elevados na medida em que o indivíduo envelhece"(5).

Levando em consideração a superpopulação de idosos e a importância do controle e prevenção de doenças nesta faixa etária, citada no texto anteriormente, pelo Ministério da Saúde e por meio da Política Nacional do Idoso, torna-se evidente a significância do problema e sua repercussão sócioeconômica, vindo a refletir em todos os níveis de atenção à saúde. Em nosso estudo daremos ênfase ao trabalho realizado por enfermeiros junto a idosos diabéticos na atenção primária à saúde.

A consulta de enfermagem, instrumento para o cuidado de enfermagem, apresenta-se como direcionadora das ações dispensadas ao cliente, estando fundamentada na necessidade de cientificidade das ações desenvolvidas. Supõe a entrevista para coleta dos dados, o exame físico, o estabelecimento do diagnóstico de enfermagem, a prescrição, estabelecimento de resultados esperados, a implementação dos cuidados e a orientação das ações relativas aos problemas encontrados ${ }^{(6)}$.

A Lei n. 7.498, de 25 de Junho de 1986, referente ao Exercício Profissional de Enfermagem fornece respaldo legal para o desenvolvimento da Consulta de Enfermagem, entendida como uma das atividades que melhor caracteriza a prática liberal da categoria ${ }^{(7)}$.

A pesquisa em questão, foi baseada nas normas emitidas pelo Ministério da Saúde ${ }^{(8-9)}$ para o cuidado ao envelhecimento, atenção à saúde da pessoa idosa e para o portador de DM. Ambos trazem informações detalhadas de todos os cuidados que devem ser prestados à clientela idosa e diabética, inclusive as ações específicas de cada profissão.

Considerando o que foi exposto, nos propusemos a descrever as ações que os enfermeiros realizam junto aos idosos diabéticos nas Unidades 
Básicas de Saúde, e avaliá-las a partir das ações preconizadas por normas técnicas do Ministério da Saúde.

\section{MATERIAL E MÉTODOS}

Trata-se de um estudo transversal de caráter descritivo, realizado durante os meses de setembro e outubro de 2008, nos Centros de Saúde que possuíam equipes da Estratégia Saúde da Família-ESF.

As unidades foram selecionadas por amostra aleatória simples, por meio de sorteio a partir de listagem ordenada das mesmas. Foram incluídas no sorteio unidades que haviam implantado o Programa de Controle de Diabetes das Secretarias Executivas Regionais-SER III e IV do município de Fortaleza-CE.

A população do estudo era composta por 52 enfermeiros que integravam as equipes de ESF, destes 42 compuseram a amostra da pesquisa, escolhidos por conveniência, convidados a participar do estudo nos dias do comparecimento dos pesquisadores às unidades de saúde.

A coleta dos dados ocorreu em dois momentos. No primeiro, foi utilizado um questionário com questões estruturadas, abordando temas relacionados aos dados de identificação e perfil profissional (qualificação, meios de atualização na área do idoso e Diabetes Mellitus), juntamente com as atividades de enfermagem realizadas nos postos de saúde à clientela idosa diabética.

No segundo momento, realizou-se observação não participante, na qual o observador permanece de fora do fenômeno, não participando dele; ele atua como expectador. A observação não participante é utilizada quando se deseja fazer uma observação naturalística, tendo em vista que não se objetiva que o observador interfira no fenômeno investigado ${ }^{(10)}$. Nessa etapa foi utilizado um roteiro de observação (checklist) elaborado pelos autores conforme manuais do Ministério da Saúde, voltado para as ações do enfermeiro na consulta aos pacientes idosos e diabéticos atendidos nas unidades básicas de saúde.

Para tabulação e análise dos dados utilizou-se o software SPSS 11.5 e, posteriormente, foi utilizada análise descritiva, para caracterizar a amostra com respaldo em literatura específica relacionada à temática do estudo.

O estudo seguiu as normas da Resolução n. 196/ 96 do Conselho Nacional de Saúde, Ministério de Saúde, que trata de pesquisas envolvendo seres Humanos, sendo iniciado após aprovação pelo Comitê de Ética em Pesquisa da Universidade Federal do Ceará, obtendo o número de Protocolo COMEPE 134/ 0.

\section{RESULTADOS E DISCUSSÃO}

\section{Perfil dos participantes do estudo}

Participaram do estudo 42 enfermeiros, dos quais $41(97,6 \%)$ eram do sexo feminino. No tocante à faixa etária, 19 (45,2\%) participantes possuíam idades compreendidas entre 31-40 anos. Quanto ao estado civil 22 (52,4\%) eram casados. Em relação ao tempo de formação profissional, 6 (14,3\%) tinham entre 1 e 5 anos, 14 (33,3\%) tinham entre 6 a 10 anos, 12(28,6\%) tinham entre $11-15$ anos, $1(2,4 \%)$ tinham entre 16-20, 8 (19\%) tinham mais de 21 anos de formação e $1(2,4 \%)$ não informou. Quanto ao período que trabalhavam na unidade de saúde, 36 (85,7\%), tinham entre 1-10 anos de serviço e 3 (7,1\%) trabalhavam há mais de 30 anos na unidade de saúde.

Com a implantação do Programa Saúde da Família em 1994, atualmente denominada Estratégia Saúde da Família-ESF, as estruturas curriculares das universidades inseriram disciplinas voltadas para atividades de prevenção e promoção da saúde da população de acordo com preceitos da estratégia.

Em nosso estudo observamos uma prevalência de enfermeiros que tiveram a formação profissional após o período de criação do ESF, 1994 e, portanto, apresentavam essa concepção de trabalho, pois, tiveram a oportunidade de vivenciar o modelo de trabalho do Programa Saúde da Família em sua graduação. Enquanto os que se formaram anteriormente a esse período, entre 11 e 15 anos, constituiram $12(28,6 \%)$ a segunda maior parcela.

Quanto à qualificação dos profissionais participantes da pesquisa observou-se que 41 (97,6\%) haviam realizado capacitação profissional e 29 (69\%) deles tinham especialização. Os que participaram de capacitação em serviço foram: 21 (50\%) em atenção básica, 12 (28,6\%) em cuidados a pacientes portadores de DM, 8 (19\%) em saúde do idoso, 1 não tinha nenhum tipo de capacitação; 24 (57,1\%) em outras áreas de atenção à saúde. A capacitação é definida como um meio de habilitar um profissional a realizar determinada função, frequentemente é realizada durante um período de 40 horas.

Nenhum dos profissionais informou ter especialização em gerontologia e 29 (69\%) dos 
enfermeiros informaram possuir especialização em saúde da família. Apesar de identificarmos um déficit na qualificação específica para atendimento a idosos diabéticos, a especialização em saúde da família apresentou-se como sendo a mais cursada pelos profissionais. Esta inclui como conteúdo, disciplinas de saúde do idoso e atendimento ao cliente diabético.

Em relação aos principais meios de atualização na área de diabetes e de cuidado a idosos, 28 (66,7\%) utilizam a internet como meio de atualização, 24 (57,1\%) utilizam livros, 39 (92,9\%) atualizam-se por Manuais do Ministério da Saúde-MS, 16 (38,1\%) participam de cursos nas áreas, 11 (26,2\%) participam de eventos na área, como congressos, seminários e encontros , e 1 (2,4\%) não utiliza nenhum meio de atualização nas áreas.

Destaca-se o elevado número de profissionais que recorrem aos Manuais do Ministério da Saúde para se atualizar. Os Manuais são fornecidos às unidades de saúde de todo o país como meio de orientar os profissionais a realizar importantes ações de combate, controle e prevenção de diversas patologias, assim como, promover uma correta assistência a uma clientela específica e significativa, por exemplo, idosos e diabéticos.

Dessa forma, os conteúdos científicos destes manuais devem ser utilizados como norteadores para a prática do enfermeiro, uma vez que fornecem protocolos ou sugestões de ações, com tópicos sucintos. Seu uso não dispensa o estudo mais aprofundado dos livros e textos de cada especialidade.

Os livros, ao contrário dos manuais, trazem informações mais fundamentadas e direcionadas aos enfermeiros. A internet também foi muito apontada como meio de atualização pelos profissionais.

Atualmente há uma tendência ao uso de tecnologias avançadas na área de educação. A Internet tem sido citada como uma das principais ferramentas de ensino, devido à numerosa quantidade de informação que está disponível na rede. Informação esta que pode estar apresentada por meio de som, imagem e texto escrito ${ }^{(11)}$.

Devemos ressaltar que a consulta à internet exige critério no que diz respeito à confiabilidade das informações, pois, diversos sites divulgam informações desatualizadas, sem cunho científico e voltadas para um público leigo.

As participações em eventos na área gerontológica foram pouco citadas. O Ministério da Saúde ${ }^{(9)}$ enfatiza a importância da promoção de uma educação permanente dos profissionais de saúde de todas as categorias. Esta exigência não é irrelevante, já que a área da saúde é constantemente aperfeiçoada conforme a realização de estudos e definição de parâmetros como, por exemplo, os valores de glicemia para o diagnóstico de DM são modificados à medida que os resultados dos estudos trazem novas evidências.

Na identificação dos meios utilizados pelos profissionais para a busca de novos casos de idosos diabéticos nas UBS, constatou-se que $41(97,6 \%)$ dos enfermeiros realizam a busca ativa de novos casos de diabéticos, incluindo idosos. A busca ativa é uma ação estratégica mínima da atenção básica para controle do DM.

O meio de busca mais prevalente foi por informação colhida pelo Agente Comunitário de SaúdeACS por meio da Ficha B-DIA ${ }^{(12)}$. Esta ficha serve para o cadastramento e acompanhamento mensal dos diabéticos com diagnóstico médico estabelecido, os casos suspeitos devem ser encaminhados à Unidade de Saúde para a realização da consulta médica ${ }^{(13)}$.

É importante salientar que os Agentes Comunitários da Saúde-ACS que realizam tal atividade devem ser acompanhados pelo enfermeiro responsável. A cada cadastro de um novo caso de diagnóstico médico de diabetes, o ACS deve informar ao enfermeiro supervisor ou outro profissional da saúde para a realização do acompanhamento do mesmo.

Quanto à busca de novos casos durante a consulta a idosos, sabe-se que ainda não é uma realidade nos serviços de saúde um atendimento específico a esta clientela, apesar de apresentar-se como sendo a de maior demanda nas unidades de saúde. O que se faz concretamente ao idoso na unidade de saúde é o atendimento prioritário. A Lei n. 8.842, de 4 de janeiro de 1994 referente à Política Nacional do Idoso e Decreto n. 1.948, de 3 de Julho de 1996 Regulamentador da Política Nacional, foram responsáveis pelo respaldo legal quanto ao atendimento preferencial do idoso em todos os órgãos públicos e privados prestadores de serviços à população ${ }^{(14-15)}$.

\section{As atividades realizadas pelos enfermeiros na atenção básica}

A Tabela 1 apresenta as atividades identificadas como aquelas realizadas pelos enfermeiros participantes do estudo voltadas para o cuidado dos idosos com Diabetes Mellitus Tipo 2. 
Tabela 1 - Atividades de Enfermagem realizadas nas UBS com idosos diabéticos. Fortaleza- CE, 2008

\begin{tabular}{lcc}
\hline Atuação do enfermeiro & Frequência & Porcentagem (\%) \\
\hline Realiza Consulta de & 42 & 100 \\
Enfermagem & 33 & 78,6 \\
Visita Domiciliária & 34 & 81 \\
Ações Educativas & 28 & 66,7 \\
Prevenção de & & 100 \\
complicações & 42 & \\
\hline Total &
\end{tabular}

Ao analisarmos a Tabela 1, observamos que 42 (100\%) dos entrevistados realizam a consulta de enfermagem, seguida por ações educativas, com um número de 34 (81\%). É importante destacarmos que o total de atividades relatadas pelos enfermeiros e apresentadas na Tabela é um número maior que o de participantes, pois, as atividades foram por vezes citadas repetidamente por mais de um profissional.

Em nosso estudo não foram investigados os tipos de ações educativas realizadas nem o tipo de prevenção de complicações que o profissional fazia em sua unidade de saúde, sendo estas apenas apontadas pelos enfermeiros.

Essas atividades citadas na tabela acima são preconizadas pelo Ministério da Saúde e operacionalizadas pelas Normas Operacionais de Assistência à Saúde-NOAS/SUS ${ }^{(16)}$. A consulta de enfermagem, por exemplo, surgiu em nosso país na década de sessenta e sua legalização ocorreu em 25 de junho de 1986 através da Lei n. ${ }^{\circ}$ 7.498/86, que regulamentou o Exercício da Enfermagem e estabeleceu essa atividade como privativa do enfermeiro ${ }^{(7)}$.

Ela é conhecida como uma atribuição do enfermeiro para o direcionamento da assistência de enfermagem, que deverá ser prestada ao cliente, por meio da qual são identificados problemas de saúde, bem como prescritas e implementadas medidas de enfermagem que contribuam para a promoção, proteção, recuperação ou reabilitação do idoso portador de DM2.

Em nosso estudo, a consulta de enfermagem mostra-se prevalente em todas as unidades de saúde visitadas, mostrando que a oferta dessa atividade para a população idosa acometida por DM2 está sendo satisfatória.

Outro dado observado neste estudo é o menor número de atividades relacionadas às prevenções de complicações associadas à DM2, relatadas apenas por
$28(66,7 \%)$ dos enfermeiros.

O Ministério da Saúde ${ }^{(8)}$ enfatiza a importância da prevenção e dos cuidados aos idosos para possíveis complicações a longo prazo, provocado pelo DM2, principalmente relativa aos problemas oculares, vasculares e renais. Evitar a cegueira e a amputação, por meio da detecção e tratamento precoces da retinopatia e das úlceras do pé, podem significar a diferença entre a institucionalização e a continuação da vida independente para a pessoa idosa com DM2.

É necessário que a equipe de enfermagem possa realizar atividades em grupos ou individualmente com os idosos, promovendo orientações acerca da alimentação, cuidado com os pés e olhos, administração correta de insulina e hipoglicemiantes orais, tais atividades são sugeridas pelo Ministério da Saúde como sendo atribuições de responsabilidade do enfermeiro da equipe de saúde.

Desse modo, é fundamental que os enfermeiros atuantes na atenção básica compreendam o processo de envelhecimento, de forma a direcionar seus esforços para ações educativas que considerem possíveis devido às limitações do idoso, principalmente no tocante à motivação para a adesão à terapêutica ${ }^{(17)}$.

A Tabela 2 apresenta as ações relatadas pelos enfermeiros como aquelas que são realizadas durante a consulta de enfermagem aos idosos. Os dados da Tabela foram utilizados para posterior confrontação com os achados da observação não-participante.

As ações citadas na Tabela 2 são preconizadas pelo Ministério da Saúde, as quais devem ser realizadas, como protocolo, durante consulta ao idoso diabético. $\mathrm{O}$ enfermeiro deve incentivar o idoso a adquirir habilidades de autocuidado diário para evitar as flutuações agudas na glicose sanguínea, o que significa orientar sobre a incorporação de muitos comportamentos preventivos para evitar complicações da doença a longo prazo.

Durante a consulta de enfermagem é importante que os pacientes diabéticos sejam orientados pelo enfermeiro sobre dieta, efeitos colaterais dos medicamentos, prática de atividade física, progressão da doença, estratégias de prevenção, técnicas de monitoração da glicose sanguínea e ajuste da medicação.

Com a educação dos portadores de diabetes, é possível conseguir reduções importantes das complicações da doença e consequente melhoria da qualidade de vida ${ }^{(15)}$.

O que é possível observar na Tabela 2 é que 
muitos profissionais limitam-se a estas ações e apenas $4(9,5 \%)$ enfermeiros realizam outras atividades, como orientações sobre dieta e atividades físicas e atendimento multidisciplinar.

Tabela 2 - Ações realizadas durante a Consulta de Enfermagem

\begin{tabular}{lcc}
\hline Ações de Enfermagem & Frequência & $\begin{array}{c}\text { Porcentagem } \\
(\%)\end{array}$ \\
\hline Realiza & 41 & 97,6 \\
Orienta sobre saúde/diabetes & 37 & 88,1 \\
Identifica fatores de risco & 41 & 97,6 \\
Verifica glicemia capilar & 40 & 95,2 \\
Realiza exame físico & 25 & 59,5 \\
Solicita e avalia exames & & \\
complementares & 39 & 92,9 \\
Informa sobre complicações & 41 & 97,6 \\
Informa sobre o uso correto de & & \\
medicamentos & 41 & 97,6 \\
Incentiva adesão ao tratamento & 38 & 90,5 \\
Estabelece consulta de retorno & 39 & 92,9 \\
Realiza exame dos MMII & 29 & 69 \\
Repete a medicação de & & 95,2 \\
indivíduos controlados & 40 & 9,5 \\
Outros & 4 & 2,4 \\
Não realiza & 1 & 100 \\
\hline Total & 42 & \\
\hline
\end{tabular}

Um dos profissionais entrevistados justificou a não realização dessas ações devido ao fato do reduzido tempo para atendimento a um grande número de pacientes. Durante a observação não-participante foi possível visualizar a alta demanda nos postos de saúde, porém, essa justificativa não se mostra relevante, pois, os demais entrevistados tinham as mesmas atribuições, mas não utilizaram esta justificativa.

Observou-se que a realização de exame físico, assim como exame dos membros inferiores é feito em menor frequência em comparação com outras ações, 25 (59,5\%) e 29 (69\%) respectivamente, confirmando os dados da observação, um entrevistado realizava os exames e três entrevistados não os realizavam.

Quanto às informações sobre o uso correto dos medicamentos, observamos que a maioria dos profissionais transcrevia os medicamentos do protocolo recomendado pelo Ministério da Saúde, informavam sobre os horários corretos da administração da medicação, confirmando os dados da observação, porém, quanto ao uso da insulina, deixavam de informar sobre associações da medicação com a alimentação, os efeitos colaterais da medicação e a dosagem correta.

\section{CONSIDERAÇÕES FINAIS}

A atenção à saúde dos idosos portadores de DM2 é uma das prioridades de saúde pública, tornando necessário ampliar o conhecimento do enfermeiro sobre o envelhecimento, as doenças crônicas e os fatores que têm influenciado as condições de saúde e qualidade de vida da população idosa.

Os dados apresentados no estudo nos mostraram a pequena parcela de enfermeiros que realizaram cursos de capacitação na área de diabetes, tornando desta forma relevante destacarmos a importância do investimento em nível de capacitação destes profissionais da área da saúde atuantes na atenção básica, capacitando-os para atuar na prevenção de complicações do diabetes nos idosos assistidos.

Considera-se, pois, fundamental o papel do enfermeiro na promoção da saúde da população idosa diabética assistida na atenção básica de saúde através de ações que promovam e estimulem a independência, a autonomia e a melhoria da qualidade de vida dos idosos.

A atualização e a implementação de técnicas fundamentadas nos manuais propostos pelo Ministério da Saúde, são fatores fundamentais para uma assistência de enfermagem qualificada baseadas nas reais necessidades dos idosos que procuram o serviço de atenção básica.

Tendo em vista as necessidades de cuidado advindas das alterações decorrentes do DM associadas às mudanças fisiológicas do processo de envelhecimento humano evidencia-se, através dos dados apresentados, a importância do estímulo à busca do conhecimento teórico-prático na área de diabetes na parcela da população idosa, pelos enfermeiros atuantes na atenção básica de saúde e a sensibilização destes no alcance dos objetivos da prevenção de agravos, incapacidades e promoção da saúde.

Acredita-se que por meio da parceria entre os cursos de graduação e pós-graduação existentes na área da gerontologia, gestores, enfermeiros e demais envolvidos na promoção da saúde dos idosos portadores de DM2, seja possível ampliar e qualificar as ações de cuidado a essa clientela, de modo que se possa alcançar a integralidade da assistência. 


\section{REFERÊNCIAS}

1. Lenardt MH, Borghi ACS, Seima MD, Hammerschmidt KSA, Michel T. O cuidado gerontogeriátrico em unidade de tratamento hemodialítico [periódico na Internet]. Cogitare Enferm 2009 [acesso em 2009 Ago 24] 14(1): 37-43. Disponível: http://ojs.c3sl.ufpr.br/ojs2/index. php/cogitare/article/view/14100/9484.

2. Seixas CT. A enfermagem brasileira frente ao envelhecimento populacional: cenários possíveis para 2025 [dissertação]. Universidade Federal de Minas Gerais; 2007. [Acesso em 2008 Out 07]. Disponível: http:/ /www.enf.ufmg.br/mestrado/dissertacoes/ ClarissaSeixas.pdf

3. Ministério da Saúde (BR). Portaria do Gabinete do Ministro de Estado da Saúde de $n^{\circ} 1395$, de 9 de dezembro de 1999, que aprova a Política Nacional de Saúde do Idoso e dá outras providências. Diário Oficial da República Federativa do Brasil, Brasília, 13 dez. 1999. Seção 1.

4. Sociedade Brasileira de Diabetes. Consenso brasileiro sobre diabetes: diagnóstico e classificação de diabetes mellitus e tratamento do diabetes mellitus tipo 2. São Paulo: Sociedade Brasileira de Diabetes; 2002. [acesso em 2008 Nov 20]. Disponível: http://www.diabetes.org.br

5. Milech A, Peixoto MC. Quadro clínico. In: Oliveira JEP, Milech A. Diabetes mellitus: clínica diagnóstico, tratamento interdisciplinar. São Paulo: Atheneu; 2004. p.33-43.

6. Maciel ICF, Araújo TL. Consulta de enfermagem: análise das ações junto a programas de hipertensão arterial, em Fortaleza [periódico na Internet]. Rev. Latino-Am. Enfermagem. 2003 [acesso em 2008 Mai 18]11(2): 207214. Disponível: http://www.scielo.br/scielo.php?script =sci_arttext\&pid=S0104-11692003000200010.

7. Brasil. Lei n. 7.498, de 25 de junho de 1986. Dispõe sobre a regulamentação do exercício da enfermagem e dá outras providências. Diário Oficial da República Federativa do Brasil, Brasília, 26 jun. 1986. Seção 1:1. Brasil. Constituição da República Federativa do Brasil. Brasília: Senado; 1988

8. Ministério da Saúde (BR). Secretaria de Atenção à Saúde. Departamento de Atenção Básica. Diabetes Mellitus. Série A, Normas e Manuais Técnicos. Cad. 16, Brasília; 2006.

9. Ministério da Saúde (BR). Secretaria de Atenção à Saúde. Departamento de Atenção Básica. Envelhecimento e Saúde da pessoa idosa. Série A, Normas e Manuais Técnicos. Cad. 19 Brasília; 2006.
10. Lakatos EM, Marconi MA. Técnicas de pesquisa. $5^{\text {a }}$ ed. São Paulo: Atlas; 2002.

11. Garcia, HD. A terceira idade e a internet: uma questão para o novo milênio [dissertação]. Marília (SP): Universidade Estadual Paulista; 2001. [acesso em 2009 Ago 31]. Disponível: http://www.marilia.unesp.br/ Home/Pos-Graduacao/CienciadaInformacao/ Dissertacoes/dominguez_garcia_me_mar.pdf

12. Ministério da Saúde (BR). Manual do Sistema de Informação de Atenção Básica. Secretaria de Políticas de Saúde, Departamento de Atenção Básica. - Brasília: Ministério da Saúde, 2000.

13. Maldonato A, Bloise D, Ceci M, Fraticelli E, Fallucca F. Diabetes mellitus: lessons from patient education. [periódico na Internet]. Patient Educ Couns . 1995 [acesso em 2008 Mai 19] 26(1-3): 57-66. Disponível em: www.ncbi.nlm.nih.gov/pubmed/7494756

14. Brasil. Lei n. 8.842, de 4 de Janeiro de 1994 . Dispõe sobre a Política Nacional do Idoso, cria o Conselho Nacional do Idoso e dá outras providências. Diário Oficial da República Federativa do Brasil, Brasília, 3 jul. 1993. Seção 1:1. Brasil. Constituição da República Federativa do Brasil. Brasília: Senado; 1988

15. Brasil. Decreto n. 1.948, de 3 de Julho de 1996. Regulamenta a Lei ${ }^{\circ} 8.842$, de 4 de janeiro de 1994, que dispõe sobre a Política Nacional do Idoso, e dá outras providências. Diário Oficial da República Federativa do Brasil, Brasília, 3 jul. 1993. Seção 1:1. Brasil. Constituição da República Federativa do Brasil. Brasília: Senado; 1988

16. Brasil. Norma Operacional da Assistência à Saúde. NOAS-SUS 01/02 Diário Oficial da União n. 20-E, de 29 de jan de 2001, Seção 1.

17. Cazarini RP, Zanetti ML, Ribeiro KP, Pace AE, Foss MC. Adesão a um grupo educativo de pessoas portadoras de diabetes mellitus: porcentagem e causas [periódico na Internet] Rev. Medicina, Ribeirão Preto, 2002 [acesso em 2009Ago 27] 35: 142-150 Abr/Jun. Disponível: http:/ /www.fmrp.usp.br/revista/2002/vol135n2/adesao_a_um grupo_educativo1p65.pdf. 